\title{
CCPLS reveals cell-type-specific spatial dependence of transcriptomes in single cells
}

\author{
Takaho Tsuchiya ${ }^{1,2}$, Hiroki Hori ${ }^{1,3}$ and Haruka Ozaki ${ }^{1,2}$ * \\ ${ }^{1}$ Bioinformatics Laboratory, Faculty of Medicine, University of Tsukuba. Tsukuba 1-1-1 Tennodai, Tsukuba, Ibaraki 305-8577, Japan \\ ${ }^{2}$ Center for Artificial Intelligence Research, University of Tsukuba, 1-1-1 Tennodai, Tsukuba, Ibaraki 305-8577, Japan \\ ${ }^{3}$ Doctoral Program in Medical Sciences, Graduate School of Comprehensive Human Sciences, University of Tsukuba, 1-1-1 Tennodai, \\ Tsukuba, Ibaraki 305-8577, Japan
}

*To whom correspondence should be addressed.

Associate Editor: $X X X X X X X$

Received on XXXXX; revised on XXXXX; accepted on XXXXX

\begin{abstract}
Motivation: Cell-cell communications regulate internal cellular states of the cell, e.g., gene expression and cell functions, and play pivotal roles in normal development and disease states. Furthermore, single-cell RNA sequencing methods have revealed cell-to-cell expression variability of highly variable genes (HVGs), which is also crucial. Nevertheless, the regulation on cell-to-cell expression variability of HVGs via cell-cell communications is still unexplored. The recent advent of spatial transcriptome measurement methods has linked gene expression profiles to the spatial context of single cells, which has provided opportunities to reveal those regulations. The existing computational methods extract genes with expression levels that are influenced by neighboring cell types based on the spatial transcriptome data. However, limitations remain in the quantitativeness and interpretability: it neither focuses on HVGs, considers cooperation of neighboring cell types, nor quantifies the degree of regulation with each neighboring cell type.

Results: Here, we propose CCPLS (Cell-Cell communications analysis by Partial Least Square regression modeling), which is a statistical framework for identifying cell-cell communications as the effects of multiple neighboring cell types on cell-to-cell expression variability of HVGs, based on the spatial transcriptome data. For each cell type, CCPLS performs PLS regression modeling and reports coefficients as the quantitative index of the cell-cell communications. Evaluation using simulated data showed our method accurately estimated the effects of multiple neighboring cell types on HVGs. Furthermore, by applying CCPLS to the two real datasets, we demonstrate CCPLS can be used to extract biologically interpretable insights from the inferred cell-cell communications.

Availability: The R package is available at https://github.com/bioinfo-tsukuba/CCPLS. The data are
\end{abstract} available at https://github.com/bioinfo-tsukuba/CCPLS_paper.

Contact: haruka.ozaki@md.tsukuba.ac.jp

Supplementary information: Supplementary data are available at Bioinformatics online.

\section{Introduction}

Cell-cell communications regulate internal cellular states of the cell, e.g., gene expression and cell functions, and play pivotal roles in normal development and disease states (Sharpe and Pauken, 2018; Snijder and Pelkmans, 2011; Pelkmans, 2012). For example, in tumor progression, programmed cell death ligand 1 (PD-L1) is expressed in a tumor cell that binds to the programmed cell death protein 1 (PD-1) of T cells, thus regulating $\mathrm{T}$ cell gene expression and weakening the anti-tumor growth response (Shimizu et al., 2020). The PD-1 pathway inhibitors block this cell-cell communication and thus stop the growth of tumor cells (Sharpe and Pauken, 2018). Accordingly, it is essential to elucidate gene expression regulation via cell-cell communications in order to understand and contro complex multicellular systems (Sharpe and Pauken, 2018; Snijder and Pelkmans, 2011; Pelkmans, 2012).

Single-cell RNA sequencing (scRNA-seq) methods have been used to study complex multicellular systems. These technologies have revealed cell-to-cell expression variability of highly variable genes (HVGs) even in the same cell type, which is crucial in the normal development and disease states (Ben-Moshe and Itzkovitz, 2019; Satija et al., 2015; Stuart et al., 2019). Nevertheless, the regulation on cell-to-cell expression variability of 
HVGs via cell-cell communications has not been well characterized (BenMoshe and Itzkovitz, 2019; Satija et al., 2015; Stuart et al., 2019). Instead of the expression of HVGs, the expression of ligand and receptor genes have been the focus of the computational tools to infer potential cell-cell communications using scRNA-seq data: these tools compare expression levels of ligand and receptor genes between cell-type pairs (Armingol et al., 2020; Hou et al., 2020; Nagai et al., 2021). However, such approaches have several limitations regarding incompleteness of knowledge on the ligand-receptor pairs, potential crosstalks among ligands and receptors, and unavailability of spatial contexts of cells (Armingol et al., 2020 Hou et al., 2020; Nagai et al., 2021). Moreover, the ligand-receptor expression approach cannot directly provide insights into the effects of cell-cell communications in most of the HVGs in the same cell type. It is desirable to focus on the regulation on cell-to-cell expression variability of HVGs via cell-cell communications.

As another remarkable aspect, intracellular gene expression regulation is a multiple-input and multiple-output (MIMO) system, and a quantitative understanding is of critical importance (Akimoto et al., 2013; Janes et al., 2005). The regulation via cell-cell communications can also be considered a MIMO system, in which the degree of neighboring cell-type existence regulates the intracellular gene expression values. As an example of the PD-1 pathway, antigen-presenting cells and the tumor cells cooperatively regulate the gene expression of $\mathrm{T}$ cells, and the balance of such regulation is crucial to cell fate decisions (Sharpe and Pauken, 2018). It is essentia to develop computational methods for estimating such a MIMO system of cell-cell communications based on spatial transcriptome data.

The recent advent of spatial transcriptome measurement methods has linked gene expression profiles to the spatial context of single cells which has provided opportunities to reveal the regulation on cell-tocell expression variability of HVGs via cell-cell communications (Cho et al., 2021; Eng et al., 2019; Marx, 2021). Nevertheless, there is no computational methods based on spatial transcriptome data for estimating regulation on cell-to-cell expression variability of HVGs as a MIMO system of cell-cell communications (Armingol et al., 2020; Arnol et al., 2019; Dries et al., 2021a; Dries et al., 2021b; Longo et al., 2021; Svensson et al., 2018; Zhu et al., 2021), which could limit quantitativeness and interpretability. For example, Giotto findICG extracts genes with expression levels that are influenced by the neighboring cell types; however, it neither considers the cooperation of neighboring cell types no quantifies the degree of regulation with each neighboring cell type (Dries et al., 2021b). Other existing methods focus on the spatial gene expression variability itself and do not focus on the effect of neighboring cell types on gene expression variability (Armingol et al., 2020; Arnol et al., 2019; Dries et al., 2021a; Dries et al., 2021b; Longo et al., 2021; Svensson et al., 2018; Zhu et al., 2021). Moreover, these tools do not explicitly focus on HVGs. Thus, there is a need to develop methods to estimate the MIMO system of cell-cell communications that focus on HVGs.

Here, we propose CCPLS (Cell-Cell communications analysis by Partial Least Square regression modeling), which is a statistical framework for identifying the MIMO system of cell-cell communications, i.e., regulation on cell-to-cell expression variability of HVGs by multiple neighboring cell types, based on the spatial transcriptome data at a singlecell resolution. CCPLS performs PLS regression modeling for each cell type and reports the estimated coefficients as the quantitative index of the cell-cell communications from each neighboring cell type. Evaluation using simulated data showed our method accurately estimated the effects of multiple neighboring cell types on HVGs. Furthermore, by applying CCPLS to the two real datasets, we demonstrate CCPLS can be used to extract biologically interpretable insights from the inferred cell-cell communications.

\section{Materials and Methods}

\subsection{Problem definition}

Spatial transcriptome data at a single-cell resolution consist of a gene expression matrix, a coordinate matrix, and a cell-type label vector. Let $N$ be the number of cells, $G$ be the number of genes, and $M$ be the number of cell types. The gene expression matrix $\mathbf{Y} \in \mathbb{R}^{N \times G}$ contains the expression value $y_{i, g}$ for each cell $i(1 \leq i \leq N)$ and gene $g$ ( $1 \leq g \leq G)$. The coordinate matrix $\mathbf{P} \in \mathbb{R}^{N \times 2}$ contains the twodimensional spatial coordinate of cells $\left(p_{i, 1}, p_{i, 2}\right)(1 \leq i \leq N)$. The cell-type label vector $\mathbf{L}=\left\{l_{i} \mid l_{i} \in\{1, \ldots, M\}\right\}$ contains the cell-type label $l_{i}$ of the $M$ unique cell types. We assume the existence of highly variable genes (HVGs) specific to cell type $m(1 \leq m \leq M)$, i.e., $\mathbf{h}^{(m)}$, which is estimated from the expression matrix $\mathbf{Y}^{(m)}$ containing cells $i$ $\left(1 \leq i \leq N^{(m)}\right)$ of cell type $m$ (Stuart et al., 2019; Hafemeister and Satija, 2019)

CCPLS aims to extract HVGs $\mathbf{h}^{(m)}$ whose expression values are significantly explained by a linear sum of the effects by neighboring cell types $f$ as follows:

$$
y_{i, h}^{(m)}=\sum_{f} x_{i, f}^{(m)} w_{f, h}^{(m)}+\epsilon_{i, h}
$$

where $h \in \mathbf{h}^{(m)}, w_{f, h}^{(m)}$ denotes coefficient, $x_{i, f}^{(m)}$ denotes neighboring cell-type score, and $\epsilon_{i, h}$ is the residue term.

Simultaneously, CCPLS aims to estimate the coefficient $w_{f, h}^{(m)}$ as the direction and degree of regulation via cell-cell communications by neighboring cell types $f$ for each HVG $h$ in a manner specific to cell type $m$.

There are two key assumptions of CCPLS: (i) the cell-cell communications are composed of the linear sum of the effects of neighboring cell types; (ii) the effects of neighboring cell types are the same within each cell type.

\subsection{CCPLS}

\subsubsection{Methods overview}

The core of CCPLS is PLS regression modeling performed by setting HVG expression values $y_{i, h}^{(m)}$ as responsive variables and neighboring cell-type scores $x_{i, f}^{(m)}$ as explanatory variables for the each cell type $m$. After the statistical evaluation, CCPLS performs HVGs $\mathbf{h}^{(m)}$ clustering, which is reported with filtered coefficients as the direction and degree of regulation in the cell-cell communication. The input data for CCPLS are the expression matrix $\mathbf{Y}$, the coordinate matrix $\mathbf{P}$, and the cell-type label vector $\mathbf{L}$. CCPLS consists of six main steps as described in sections 2.2.2-2.2.7, respectively.

Except for the calculation of neighboring cell-type scores, CCPLS is principally based on existing procedures. In other words, by calculating the neighboring cell-type scores, CCPLS realizes a multiple regression approach for estimating the MIMO system of cell-cell communications.

A schematic illustration of the MIMO system of cell-cell communications is shown in Figure 1a, while Figure 1b illustrates the workflow of CCPLS

\subsubsection{Step (i): Extraction of HVG}

CCPLS divides the input expression matrix $\mathbf{Y}$ into each cell type $\mathbf{Y}^{\prime(m)}$ the expression matrix comprised of cells $i$ for each unique cell type $m$ CCPLS filters out the genes with an expression value of 0 in all the cells within cell type $m$ from $\mathbf{Y}^{\prime(m)}$, which is subsequently normalized and for each transformed gene z-score. Simultaneously, CCPLS extracts cell-type- $m$-specific $\mathrm{HVG} \mathrm{h}^{(m)}$ and preprocessed HVG expression value $y_{i, h}^{(m)}(h=1, \ldots, H)$ and matrix $\mathbf{Y}^{(m)} \in \mathbb{R}^{N^{(m)} \times H}$ (Hafemeister and Satija, 2019; Stuart et al., 2019). 
$\mathrm{a}$

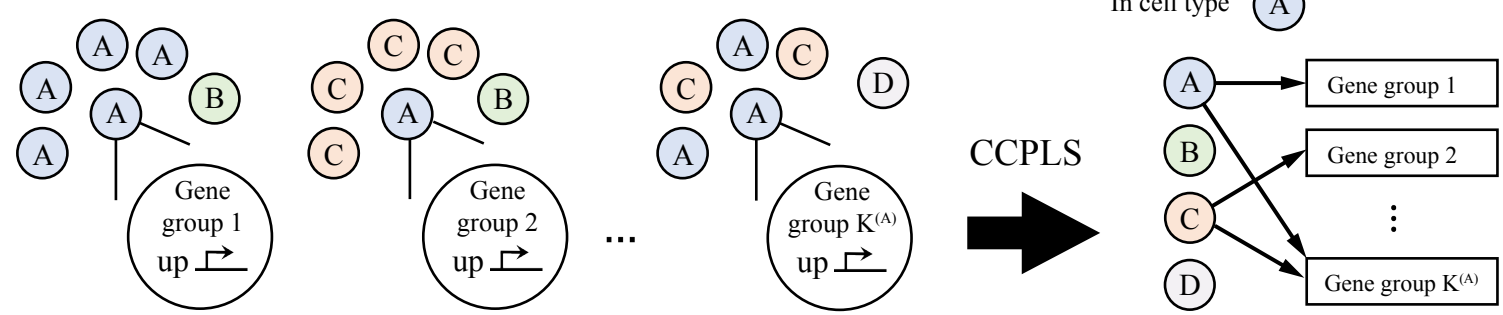

b Gene expression matrix $Y$
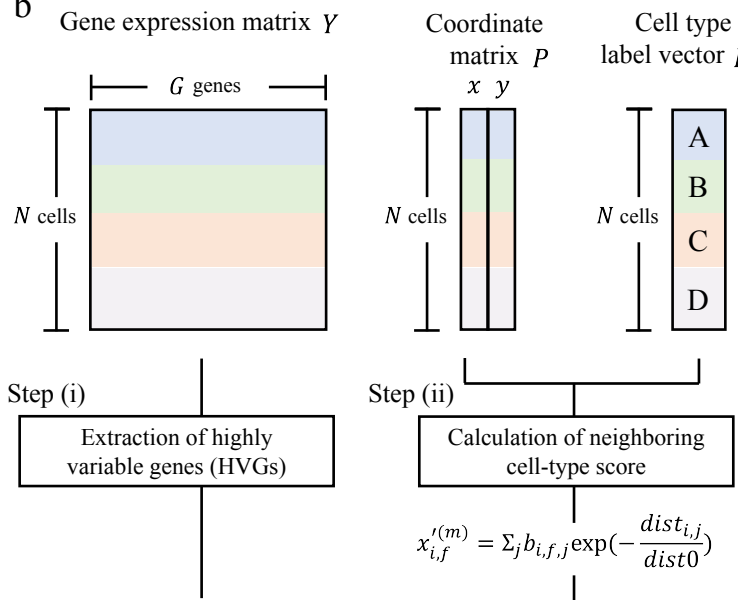
cell-type score

z score transformation for each HVG $h$

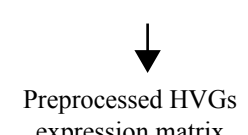

$$
x_{i, f}^{\prime(m)}=\Sigma_{j} b_{i, f, j} \exp \left(-\frac{\text { dist }_{i, j}}{\text { dist } 0}\right)
$$

$\mathrm{z}$ score transformation for each neighboring cell type $f$ expression matrix

Preprocessed neighboring cell-type score matrix
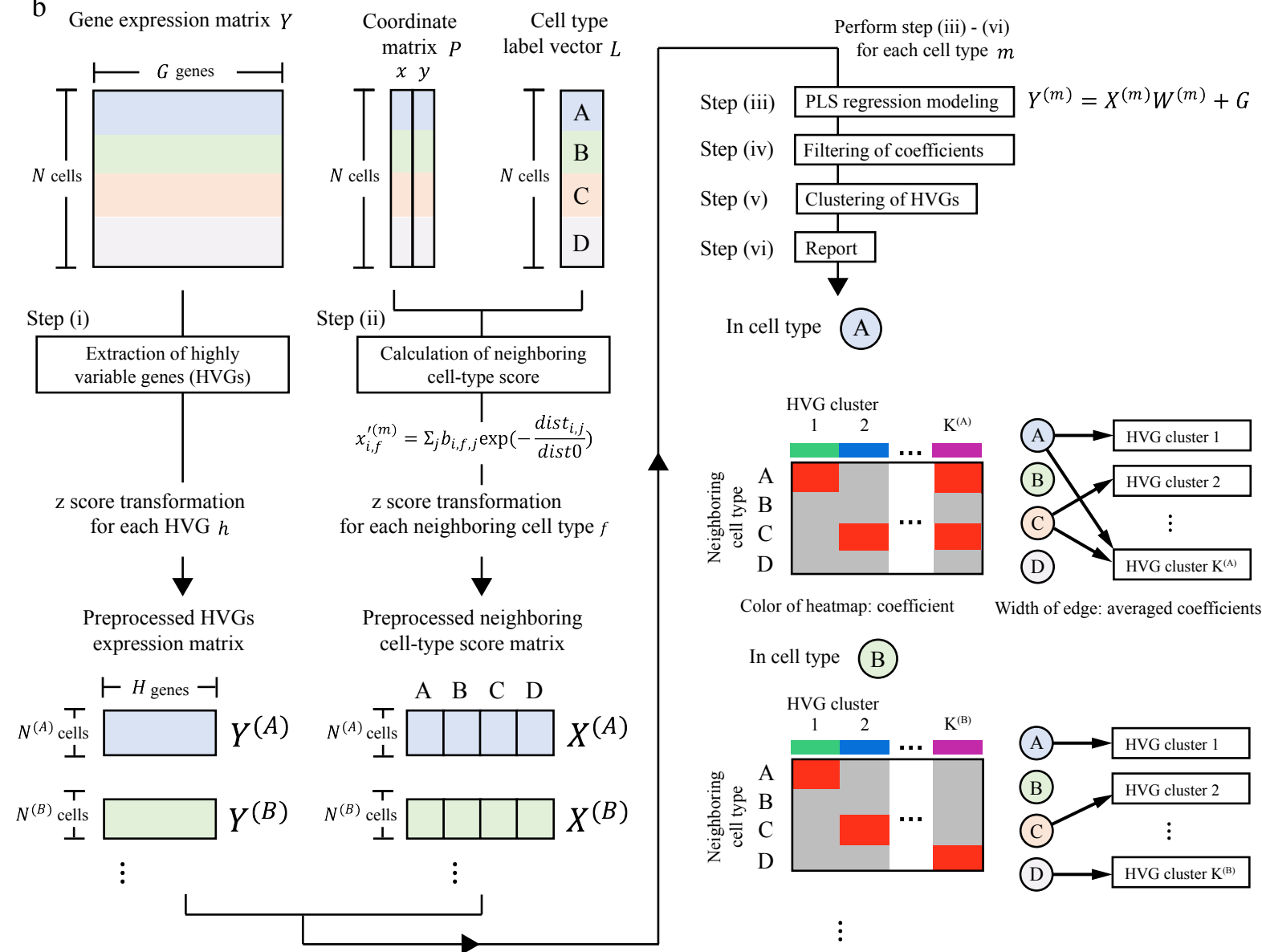

In cell type A
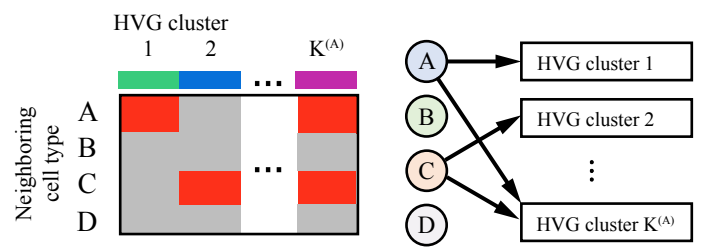

Color of heatmap: coefficient

Width of edge: averaged coefficients

$$
\text { In cell type B }
$$
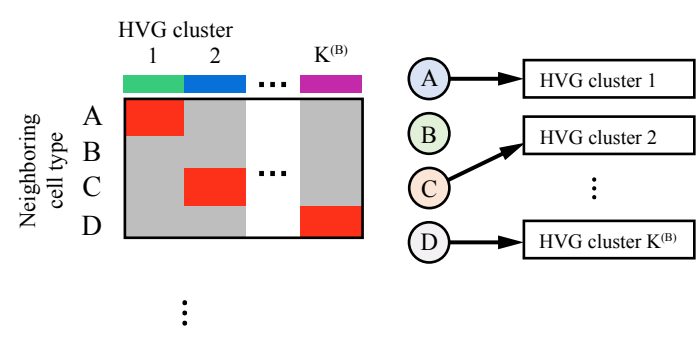

Fig. 1. Overview of CCPLS. (a) Schematic illustration of the multiple-input and multiple-output (MIMO) system of cell-cell communications that CCPLS aims to identify. (b) Workflow of CCPLS.

2.2.3 Step (ii): Calculation of neighboring cell-type score

For each cell type $m$, CCPLS calculates the degree of neighboring celltype existence $x_{i, f}^{(m)}$, denoted as the neighboring cell-type score, by using the coordinate matrix $\mathbf{P}$ and cell-type label vector $\mathbf{L}$. CCPLS calculates un-preprocessed neighboring cell-type score $x_{i, f}^{\prime(m)}$ based on a function that decays with distance between two cells $i$ and $j$ as follows:

$$
x_{i, f}^{\prime(m)}=\sum_{j} b_{i, f, j} \exp \left(-\frac{d i s t_{i, j}}{d i s t 0}\right),
$$

where the $b_{i, f, j}$ is the binary value indicating whether the neighboring cell type $l_{j}$ of cell $j(1 \leq j \leq N)$ around cell $i$ belongs to cell type $f$, and the dist $_{i^{\prime}}{ }_{j}$ is the Euclidean distance calculated from the coordinates of the two cells $i$ and $j$. The value of $d^{2} t_{0}$ is the constant set as the minimum value of the Euclidean distance of all the combinations of two cells in the coordinate matrix $\mathbf{P}$. CCPLS performs z-score transformation on the unpreprocessed neighboring cell-type score $x_{i, f}^{\prime(m)}$ for each neighboring cell type $f$ and calculates the preprocessed neighboring cell-type score matrix $\mathbf{X}^{(m)}$.

\subsubsection{Step (iii): PLS regression modeling}

For each cell type $m$, CCPLS performs PLS regression modeling (Höskuldsson, 1988). CCPLS sets the preprocessed HVG expression matrix $\mathbf{Y}^{(m)}$ as response variables and the preprocessed neighboring celltype score matrix $\mathbf{X}^{(m)}$ as explanatory variables and then estimates the coefficient matrix $\mathbf{W}^{(m)}$ as follows:

$$
\mathbf{Y}^{(m)}=\mathbf{X}^{(m)} \mathbf{W}^{(m)}+\mathbf{G}
$$


Table 1. Coefficients $w_{f, h}^{(A)}$ in the simulation experiments where $\mathbf{G}$ is the residue matrix. This coefficient matrix $\mathbf{W}(m)$ consist of each coefficient $w_{f, h}^{(m)}\left(1 \leq f \leq M, h \in\left\{\mathbf{h}^{(\mathbf{m})}\right\}\right)$, which is the direction and degree of regulation in the cell-cell communications.

The optimum number of components in PLS regression modeling is determined by minimizing the mean squared error (MSE) of 10-fold crossvalidation (Bengio and Grandvalet, 2004).

\subsubsection{Step (vi): Filtering of coefficients}

For each cell type $m$, CCPLS performs two-step filtering, a $t$-test of factor loadings, and a non-parametric test of the coefficients $w_{f, h}^{(m)}$ (Yamamoto et al., 2014). In each step, the $p$-values are false discovery rate (FDR)-adjusted by the Benjamini-Hochberg (BH) method, respectively (Benjamini and Hochberg, 1995). See text S1 in the Supplementary Information for the detailed procedures.

\subsubsection{Step (v): Clustering of HVGs}

For each cell type $m$, CCPLS performs HVG clustering by the k-mean method on the coefficients $w_{f, h}^{\prime \prime}(m)$ (Yuan and Yang, 2019). The cluster number $k$ is determined by the Silhouette method (Yuan and Yang, 2019). The minimum and the maximum integer values of $k$ are set as 2 and 15 respectively. Before clustering, CCPLS filters out the HVGs whose filtered coefficients are all zero.

\subsubsection{Step (vi): Report}

CCPLS outputs a heat map and bipartite graph. The heat map reports the filtered coefficients for each combination of HVGs $h$ and neighboring cell types $f$. The bipartite graph visualizes the relationship between the HVG clusters and the neighboring cell types $f$, where the widths of edges are the averaged values of the filtered coefficients of each HVG cluster.

\subsection{Datasets}

\subsubsection{Simulated dataset}

For the evaluation of CCPLS performance, we first simulated a datase according to the following procedures. To realistically simulate the cell positions and ratio of cell types, our simulation experiments were based on the seqFISH+ real dataset (Eng et al., 2019).

We used the coordinate matrix $\mathbf{P}$ of the seqFISH+ real dataset. To simplify the cell types, we prepared a cell-type label vector $\mathbf{L}$ by substituting cell types A-D as the cell-type label vector of the seqFISH+ real dataset. We set four cell types as inputs and four HVG clusters as outputs, which contained 500 genes, respectively (Fig. 2a). HVG cluster 1 was a multiple-input case, while clusters 2-3 were single-input cases. HVG cluster 4 was not affected by neighboring cell types.

We simulated the preprocessed expression value of cell type A according to the equation $\dot{y}_{i, h}^{(A)}=\sum_{f} x_{i, f}^{(A)} w_{f, h}^{(A)}+\alpha e_{i, h}$. We prepared coefficient $w_{f, h}^{(A)}$ according to Table 1 , in which the coefficient is $w_{\max }$ or 0 . We calculated the preprocessed neighboring cell-type score $x_{i, f}^{(A)}$ using the prepared coordinate matrix $\mathbf{P}$ and the cell-type label vector $\mathbf{L}$. We simulated the term $e_{i, h}$ as Gaussian noise, with a mean of 0 and standard deviations of each respective HVG estimated by Mean-CV regression modeling of the seqFISH+ real dataset, which was then multiplied by the constant $\alpha$.

In section 3.1, we present the application of CCPLS to this simulated dataset, including an evaluation of the results of cell type A. See text S2 in the Supplementary Information for the detailed procedures.

\subsubsection{SeqFISH+ real dataset}

For the demonstration of CCPLS with a real dataset, we selected the seqFISH+ mouse somatosensory cortex dataset provided by Giotto (https://rubd.github.io/Giotto_site/articles/mouse_seqFISH_cortex_200914) \begin{tabular}{l|l|l|l|l}
\hline Neighboring & HVG cluster & HVG cluster & HVG cluster & HVG cluster
\end{tabular}

\begin{tabular}{c|l|l|l|l}
\hline $\begin{array}{c}\text { Neighboring } \\
\text { cell type }\end{array}$ & $\begin{array}{l}\text { HVG cluster } \\
1\end{array}$ & $\begin{array}{l}\text { HVG cluster } \\
2\end{array}$ & $\begin{array}{l}\text { HVG cluster } \\
3\end{array}$ & $\begin{array}{l}\text { HVG cluster } \\
4\end{array}$ \\
\hline \hline $\mathrm{A}$ & $w_{\max }$ & 0 & 0 & 0 \\
$\mathrm{~B}$ & 0 & $w_{\max }$ & 0 & 0 \\
$\mathrm{C}$ & $w_{\max }$ & 0 & $w_{\max }$ & 0 \\
$\mathrm{D}$ & 0 & 0 & 0 & 0 \\
\hline
\end{tabular}

(Dries et al., 2021b). This seqFISH+ dataset profiled 10,000 genes in 523 cells with 12 distinct cell types at a single-cell resolution by using a super-resolved imaging technique (Eng et al., 2019).

In section 3.2, we describe the results of applying CCPLS to this seqFISH+ real dataset. We further assigned the contributor cell types, which were the common neighboring cell types in each HVG cluster. cluster to investigate the associated biological insights. See text S2 in the Supplementary Information for the detailed procedures.

\subsubsection{Seq-Scope real dataset}

For the demonstration of CCPLS with a real dataset, we selected the SeqScope colon dataset, which can be acquired from the repository of Deep Blue Data (https://doi.org/10.7302/cjfe-wa35) (Cho et al., 2021). This SeqScope dataset profiled 10,806 genes in 4,489 cells with nine distinct cell types at a single-cell resolution by using Illumina flow-cell-based spatial barcoding techniques (Cho et al., 2021)

In section 3.3, we describe the results of applying CCPLS to this SeqScope real dataset, including the assignment of contributor cell types and GO enrichment analysis according to the same procedure referred to in section 2.3.2.

\section{Results}

3.1 CCPLS yields an accurate estimation of cell-cell communications

To evaluate CCPLS performance, we prepared simulated data with parameters based on the seqFISH+ real dataset and then applied CCPLS to this simulated data as described in Section 2.3.1 (Fig. 2a). We obtained a heat map and bipartite graph, which indicate the coefficient as the direction and degree of regulation in the cell-cell communication for each combination of HVGs and neighboring cell types by CCPLS (Fig. 2 bc). Cell types $\mathrm{C}$ and B exhibited upregulation of HVG clusters 1 and 2, respectively (Fig. 2 b-c), while cell types $\mathrm{A}$ and $\mathrm{C}$ cooperatively up-regulated HVG cluster 3 (Fig. 2b-c). Three cell types, A, B, and C, exhibited upregulation of HVG cluster 4, whose degree of cell-cell communications was relatively low (Fig. 2c).

We assigned the estimated HVG clusters 1-4 with the predefined clusters. The estimated HVG clusters 1, 2, and 3 corresponded to the predefined HVG clusters 3, 2, and 1, respectively. The estimated HVG cluster 4 was assigned as "others". Note that CCPLS did not report HVGs whose filtered coefficients were all zero, which corresponded to the predefined HVG cluster 4 in this case. Based on the cluster assignment, we calculated ten indexes for performance evaluation (Fig. 2d). All the calculated indexes for CCPLS results were greater than 0.97, which indicated that CCPLS estimated the prepared coefficients and the predefined HVG clusters with high performance. We also compared CCPLS with the existing method Giotto findICG, which was outperformed by CCPLS in this simulation experiment (Dries et al., 2021b) (Fig. 2d). In HVG cluster 1 of the multiple-input case, the performance between the two methods was most different. Note that we did not compare CCPLS We performed Gene Ontology (GO) enrichment analysis for each HVG 
In cell type $\mathrm{A}$

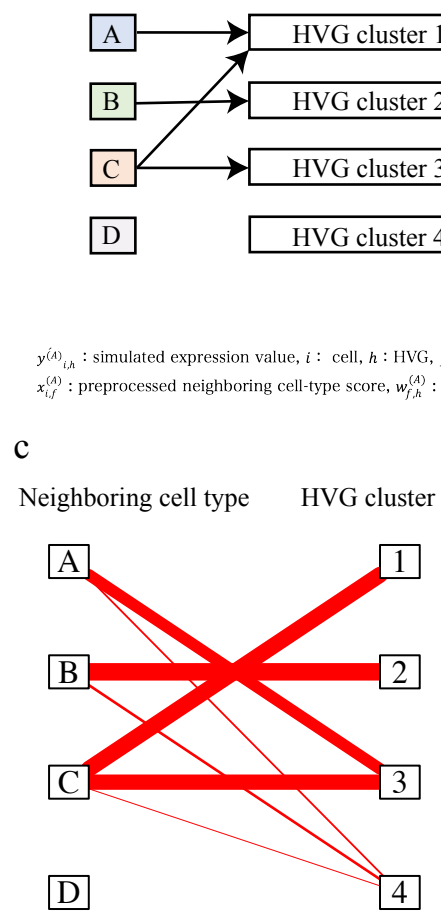

Width of edge: averaged coefficients b

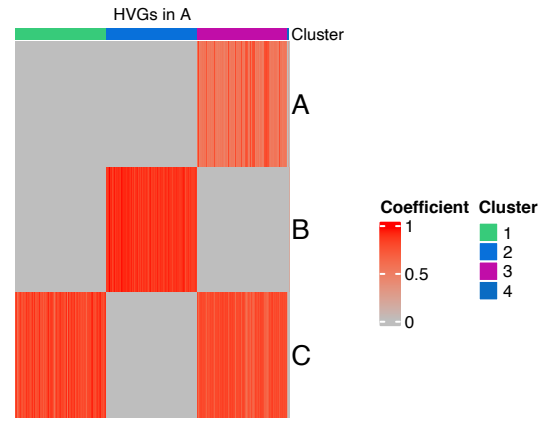

Color of heatmap: coefficient

Fig. 2. Evaluation using the simulated data. (a) Schematic illustration of the simulation settings. (b) Heat map generated by CCPLS. The color of the heat map indicates the coefficient. Rows and columns correspond to neighboring cell types and highly variable genes (HVGs), respectively. (c) Bipartite graph generated by CCPLS. The width of each edge indicates the average coefficients for each combination of HVG clusters and neighboring cell types. (d) Performance indexes and comparison with Giotto findICG. The calculated indexes are as follows: adjusted rand index, Pearson correlation coefficient, precision, and recall of each HVG cluster. Red and green indicate CCPLS and Giotto findICG results, respectively.

to the other methods, which do not estimate the effect of neighboring cell types (Table S1).

Next, we examined the performance limits of CCPLS by changing $w_{\max }$ and $\alpha$, the parameters for adjusting the degree of the cell-cell communications and Gaussian noise, respectively (Fig. S1a). According to the same procedures shown in Figure 2, we calculated the ten indexes (Fig. S1b). In each $w_{\max }$, these 10 indexes decreased when $\alpha$ increased. These ten indexes were greater than 0.97 for conditions 1-5 and 7, which indicated the estimations were successful. In conditions 6 and 8, these ten indexes were relatively lower and moderate. The estimation failed under only condition 3 , for which the eight indexes were less than 0.02 (Fig. S1b).

We further investigated the difference between the failed and successful conditions by calculating the index of variance proportion $(V P)$ as the ratio of the variance derived from the regression model term not but the Gaussian noise term. In each $w_{\max }, V P$ decreased when $\alpha$ increased, which indicated that the higher $\alpha$ conditions were more severe, such that the variance associated with cell-cell communications was more difficult to distinguish from noise (Fig. S1c). The $V P$ of the failed conditions were less than 0.04 , while the $V P$ of the successful conditions were greater than 0.61 . The estimation did not work well under excessively severe $V P$ conditions, while CCPLS estimated the prepared coefficients and the predefined HVG clusters with high performance in the relatively higher $V P$ cases. Note that CCPLS did not estimate any HVGs and cell-cell communications from randomly generated data (data not shown).

These simulation experiments showed that CCPLS yielded an accurate estimation of the MIMO system of cell-cell communications.
3.2 Application to seqFISH+ real dataset reveals biologically consistent insights associated with oligodendrocyte differentiation

To demonstrate CCPLS with a real dataset, we applied CCPLS to the seqFISH+ real dataset (Eng et al., 2019; Dries et al., 2021b). Procedures were as described in Section 2.3.2. Before we applied CCPLS, we examined the overlap of the extracted HVGs among all 12 of the cell types in the seqFISH+ real dataset (Fig. 3b). The mean value of the ratio of the overlapping HVGs was 0.25 . The majority of the HVGs were different in each comparison, which indicated that the HVGs were uniquely characterized for each cell type.

To estimate the cell-cell communications associated with these HVGs for each cell type, we applied CCPLS. We obtained the HVGs and the cellcell communications for six cell types out of all twelve cell types (Fig. 3c-d and S2-3). Focusing on the oligodendrocyte precursor cells (OPCs), two HVG clusters were estimated (Fig. 3c-d). We assigned the contributor cell types, which indicated that three cell types (astrocytes, Olig, and OPCs) cooperatively up-regulated HVG clusters 1 and 2 in the OPCs.

In addition, we performed GO enrichment analysis to investigate associated biological insights. The GO enrichment analysis was performed on the mixed gene list of HVG clusters 1 and 2, as HVG clusters 1 and 2 have the same contributor cell types.

The GO enrichment of HVG clusters 1 and 2 in OPCs indicated the 25 enriched GO terms (Fig. S5). The top $10 \mathrm{GO}$ terms are indicated in Figure 4e (Fig. 3e). GO terms related to differentiation were enriched, such as "glial cell differentiation," indicating that differentiation of OPCs 
$\mathrm{c}$ $\mathrm{a}$ Application to seqFISH+ real dataset:

Mouse somatosensory cortex

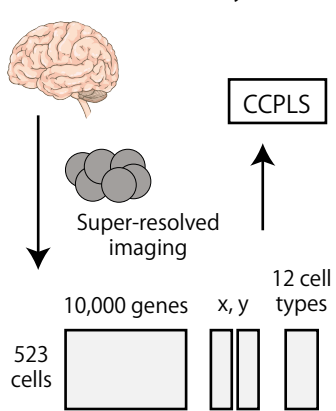

d

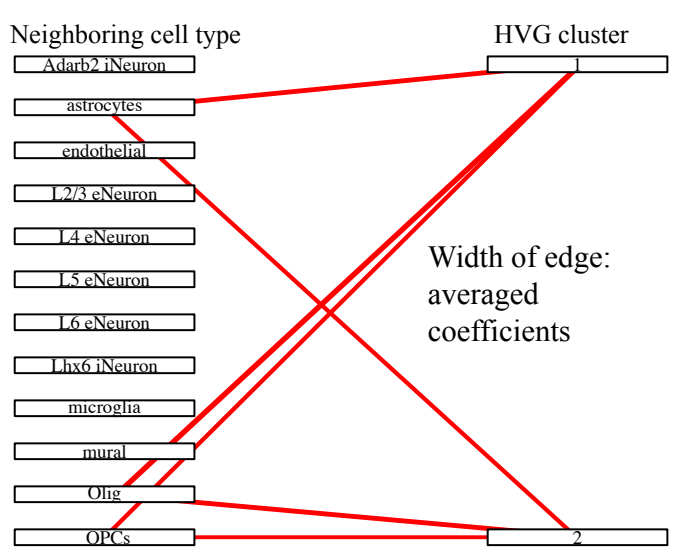

b

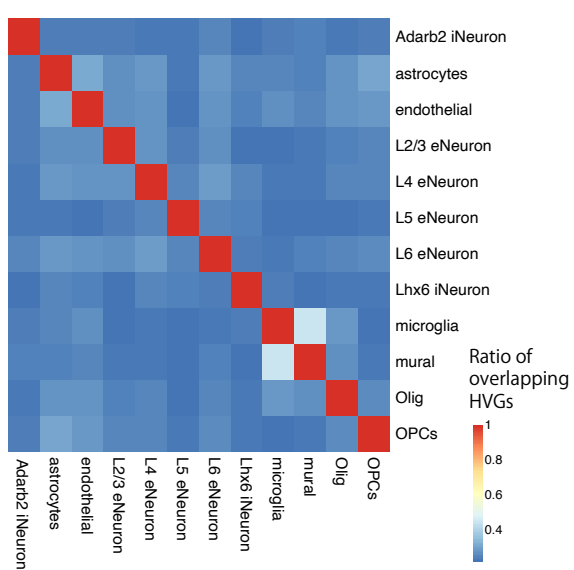

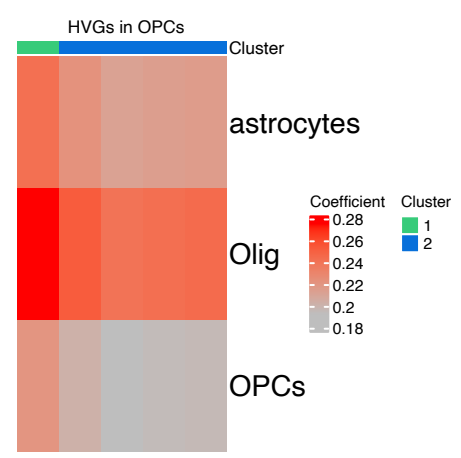

Color of heatmap: coefficient e

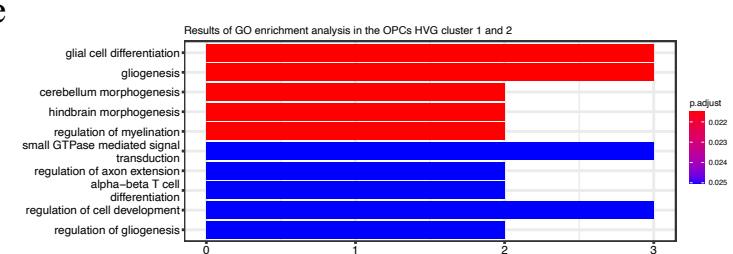

f

Oligodendrocyte differentiation communicating with astrocyte:

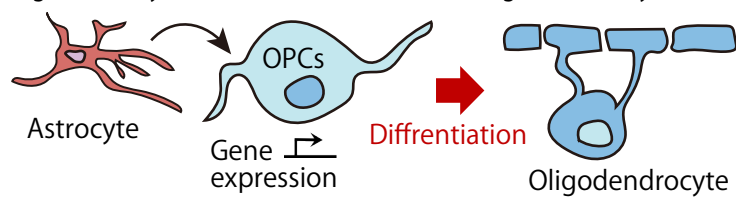

Fig. 3. Application to the seqFISH+ real dataset. (a) Schematic illustration of the seqFISH+ dataset. (b) Overlap of highly variable genes (HVGs) between cell types. Rows and columns both correspond to cell types. The color of the heat map indicates the ratio of the overlapping HVGs. (c) Heat map of oligodendrocyte precursor cells (OPCs) generated by CCPLS. Rows correspond to neighboring cell types. Columns correspond to HVGs. The color of the heat map indicates the coefficient. (d) Bipartite graph of OPCs generated by CCPLS. The width of the edge indicates the averaged coefficients for each combination of HVG clusters and neighboring cell types. (e) Gene Ontology (GO) enrichment of HVG clusters 1 and 2 for OPCs. The top $10 \mathrm{GO}$ terms are indicated. (f) Schematic illustration of the obtained biological insights on OPCs.

was induced by communicating with astrocytes, Olig, and OPCs itself. The heat map, bipartite graph, contributor cell type assignment, and GO enrichment analysis results for all cell types are shown in the Supplementary Information (Fig. S2-5, respectively). This application to the seqFISH+ real dataset indicated the capability of CCPLS to extract biologically interpretable insights.

\subsection{Application to Seq-Scope real dataset suggests epithelial cell development in immature B cell}

For the further demonstration of CCPLS using a real dataset, we applied CCPLS to the Seq-Scope real dataset (Cho et al., 2021). The procedures followed are as described in Section 2.3.3. Before we applied CCPLS, we examined the overlapping of the extracted HVGs between all nine cell types in the Seq-Scope real dataset (Fig. 4b). The mean value of the ratio of the overlap HVG was 0.31 . The majority of the HVGs differed between each comparison, which indicated that the HVGs were uniquely characterized for each cell type.

To estimate cell-cell communications associated with these HVGs for each cell type, we applied CCPLS. We obtained the HVGs and the cell-cell communications for eight cell types out of all nine cell types (Fig. 4c-d and S6-7). Focusing on the immature B cell, nine HVG clusters were estimated (Fig. 4c-d). We assigned the contributor cell types, which indicated that the immature B cell and the Fibroblast cell types cooperatively downregulated HVG cluster 1 in the immature B cell. In HVG clusters 2 to 9 , there was one contributing cell type associated with up-regulation. The contributor cell types in clusters 2 to 9 were immature B cell, DCSC, IgA B cell, Macrophage, Smooth Muscle, Stem, Paneth-like, and IgG B cell, respectively.

In addition, we performed GO enrichment analysis to investigate potential biological insights. The GO enrichment of cluster 4 for the immature B cell indicated, among the eight enriched GO terms, "epithelial cell development" was the most enriched term (Fig. 4e). These results suggested that epithelial cell development of the immature B cell occurred through communicating with IgG B cells (Fig. 4f). The heat map, bipartite graph, contributor cell type assignment, and GO enrichment analysis results for all the cell types are shown in the Supplementary Information, respectively (Fig. S6-9). The application to the Seq-Scope real dataset demonstrate the capability of CCPLS to extract biologically interpretable insights.

\section{Discussion}

In this study, we propose CCPLS (Cell-Cell communications analysis by Partial Least Square regression modeling), which is a statistical framework for identifying the MIMO system of cell-cell communications, i.e., gene 
a

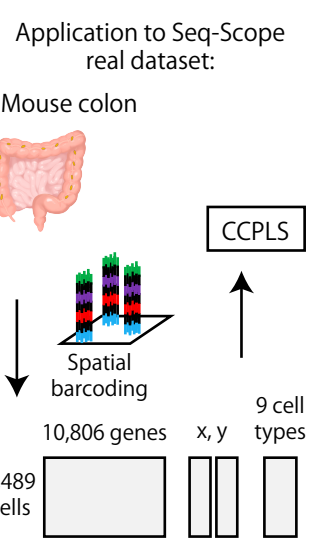

$\mathrm{d}$

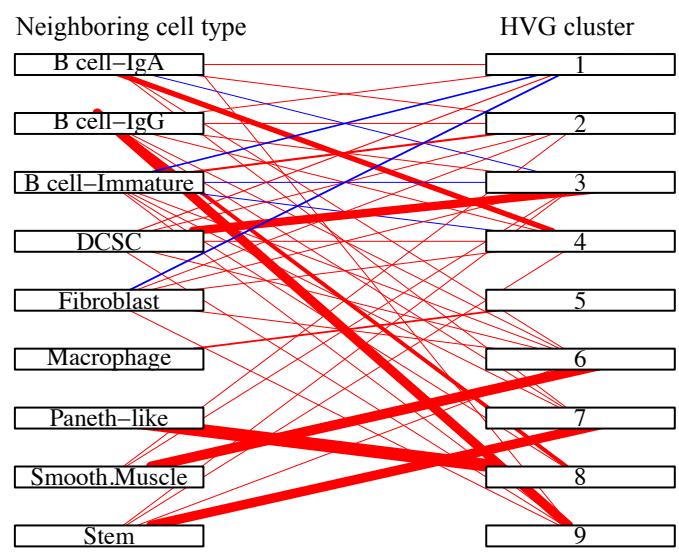

Width of edge: averaged coefficient b

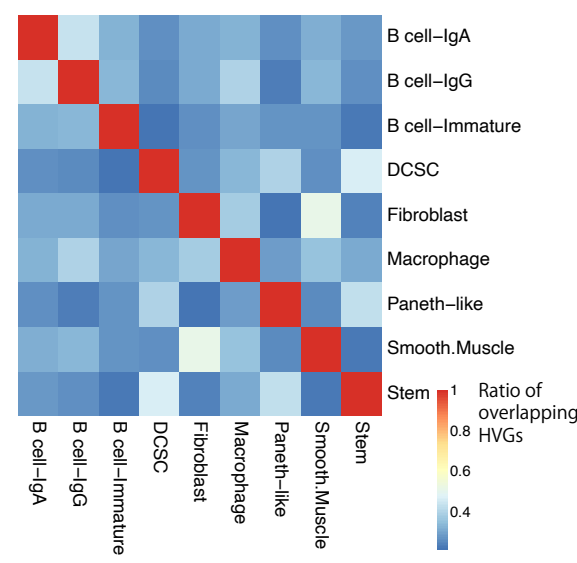

e

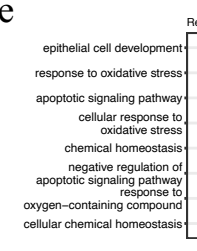

f

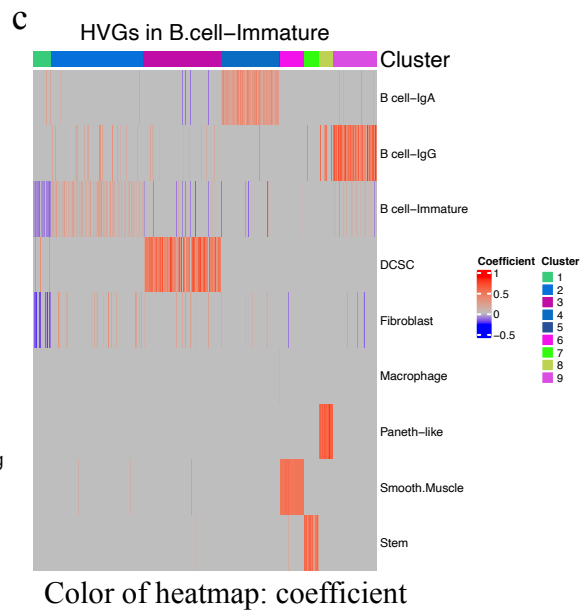

Color of heatmap: coefficient

Gene coun

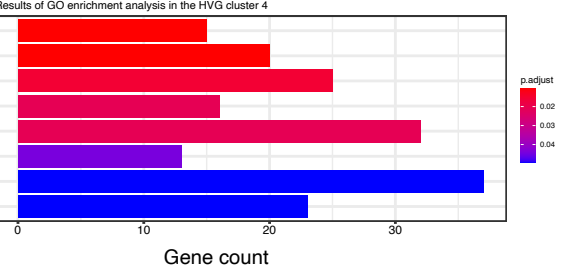
between B cells:

B cell-lgA
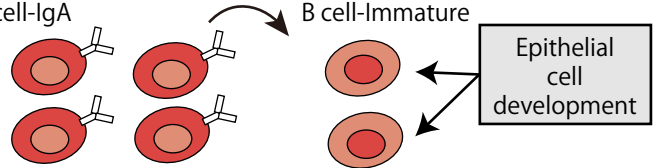

Fig. 4. Application to the Seq-Scope real dataset. (a) Schematic illustration of the Seq-Scope dataset. (b) Overlap of highly variable genes (HVGs) between cell types. Rows and column both correspond to cell types. The color of the heat map indicates the ratio of the overlapping HVGs. (c) Heat map of the immature B cell generated by CCPLS. Rows correspond to neighboring cell types. Columns correspond to HVGs. The color of the heat map indicates the coefficient. (d) Bipartite graph of the immature B cell generated by CCPLS. The width of each edge indicates the average coefficients for each combination of HVG clusters and neighboring cell types. (e) Gene Ontology (GO) enrichment of HVG cluster 4 of the immature B cell. (f) Schematic illustration of the obtained biological insights on the immature B cell.

expression regulation by multiple neighboring cell types, based on the spatial transcriptome data at a single-cell resolution. CCPLS estimates regulation on cell-to-cell expression variation of HVGs via cell-cell communications not limited to ligand-receptor pairs. The core of CCPLS is PLS regression modeling performed by setting the HVGs expression values as the responsive variables and the neighboring cell-type scores as the explanatory variables for each cell type. After the statistical evaluation, CCPLS performs HVG clustering, which is reported with coefficients as the direction and degree of regulation in the cell-cell communication. Evaluation using simulated data showed accurate estimation by CCPLS. The application of CCPLS to the two real spatial transcriptome datasets demonstrated the capability to extract biologically interpretable insights.

CCPLS differs from the existing method Giotto findICG in that CCPLS quantitatively evaluates cell-cell communications as a MIMO system by including cooperation of neighboring cell types, which may have revealed the biological insights derived from the applications to the two real datasets. In the simulation experiments, CCPLS outperformed Giotto findICG, especially with regard to HVG cluster 1, which is a multiple-input case. The application of CCPLS to the seqFISH+ real dataset showed that the differentiation in the OPCs occurred through communicating with astrocytes, Olig, and OPCs itself, which was principally consistent with previous research and not identified by Giotto findICG (Dries et al., 2021b; Nutma et al., 2020). The application to the Seq-Scope real dataset suggested that epithelial cell development is induced in the immature $B$ cell by communicating with the $\operatorname{IgG} B$ cells, which has not been reported previously to the best of our knowledge. Accordingly, further experimental follow-up studies are merited. Other existing methods, which do not focus on the effect of neighboring cell types, may have missed these findings (Armingol et al., 2020; Arnol et al., 2019; Dries et al., 2021a; Dries et al. 2021b; Svensson et al., 2018; Zhu et al., 2021). As another characteristic, CCPLS focuses on HVGs not limited to ligand-receptor pairs, which may have also enabled it to reveal the biological insights noted above.

The exploration of cell-cell communications in combination with spatial transcriptome data is still largely incomplete, and CCPLS would be effective for cell-cell communications such as those in the PD1 pathway between tumor cells and $\mathrm{T}$ cells, which led to a breakthrough drug discovery (Sharpe and Pauken, 2018). For example, the treatment of cold tumors, which are composed of tumor cells under a severe microenvironment with few neighboring $\mathrm{T}$ cells, is still considered to be a particular challenge (Haanen, 2017). Cold tumor-specific combinations of HVGs and neighboring cell types possibly cause severe tumor progression and may be candidates for drug targeting.

The limitations and perspectives in this study are as follows. CCPLS neither extracts nonlinear relationships nor estimates the cell-cell 
communications derived from factors beyond the neighboring cell-typ score, such as ligand-receptor pairs and cell-cell interactions via longrange effect (Dries et al., 2021a; Fechner and Goodman, 2018; Longo et al., 2021; Sapir and Tzlil, 2017). CCPLS does not receive as input spatial transcriptome data with a multiple-cell resolution as is characteristic of 10x Genomics Visium and Slide-seq datasets (Rodriques et al., 2019; Stickels et al., 2021; Asp et al., 2020). Expansions of CCPLS to addres these limitations are an intended focus of our future work. In addition, the responsive expression values can be replaced by the other omics data such as spatial metabolome or proteome data, which is also an intended focus of our future work (Alexandrov, 2020; Bhatia et al., 2021; Dyring-Andersen et al., 2020; Geier et al., 2020; Liu et al., 2020; Seydel, 2021).

Currently, collaborative consortiums are accumulating large-scale spatial transcriptome data (BRAIN Initiative Cell Census Network (BICCN), 2021; Zhang et al., 2021; Regev et al., 2017), and CCPLS can be readily applied to these large-scale datasets. CCPLS is provided as a package in the $\mathrm{R}$ statistical computing environment, and its calculation time was less than 10 minutes for each demonstration presented in this study. In summary, CCPLS provides novel insights into regulation on cell-to-cell expression variability of HVGs via cell-cell communications.

\section{Acknowledgements}

We thank Dr. Wataru Iwasaki, Dr. Hirotaka Matsumoto, Dr. Tsukasa Fukunaga and Dr. Kentaro Kawata for critical reading of this manuscript The computational analysis of this work was performed in part with the support of the supercomputer system of the National Institute of Genetics (NIG), Research Organization of Information and Systems (ROIS). We thank kango-roo (www.kango-roo.com) for providing illustrations.

\section{Software and data availability}

The R package is available at https://github.com/bioinfo-tsukuba/CCPLS The data are available at https://github.com/bioinfo-tsukuba/CCPLS_paper.

\section{Funding}

This work was supported by JSPS KAKENHI Grant Number 20K19915, 17H06300, 21H03124, 19H03696, and 19K20394.

Conflict of Interest: none declared.

\section{References}

Akimoto, Y. et al. (2013). The extraction of simple relationships in growth factor-specific multiple-input and multiple-output systems in cell-fate decisions by backward elimination PLS regression. PLoS One, 8(9), e72780.

Alexandrov, T. (2020). Spatial metabolomics and imaging mass spectrometry in the age of artificial intelligence. Апnи Rev Biomed Data Sci, 3, 61-87.

Armingol, E. et al. (2020). Deciphering cell-cell interactions and communication from gene expression. Nat. Rev. Genet.

Arnol, D. et al. (2019). Modeling Cell-Cell interactions from spatial molecular data with spatial variance component analysis. Cell Rep. 29(1), 202-211.e6.

Asp, M. et al. (2020). Spatially resolved Transcriptomes-Next generation tools for tissue exploration. Bioessays, 42(10), e1900221.

Ben-Moshe, S. and Itzkovitz, S. (2019). Spatial heterogeneity in the mammalian liver. Nat. Rev. Gastroenterol. Hepatol., 16(7), 395-410.
Bengio, Y. and Grandvalet, Y. (2004). No unbiased estimator of the variance of k-fold cross-validation. J. Mach. Learn. Res., 5(Sep), $1089-1105$

Benjamini, Y. and Hochberg, Y. (1995). Controlling the false discovery rate: A practical and powerful approach to multiple testing. J. R. Stat Soc., 57(1), 289-300.

Bhatia, H. S. et al. (2021). Proteomics of spatially identified tissues in whole organs.

BRAIN Initiative Cell Census Network (BICCN) (2021). A multimoda cell census and atlas of the mammalian primary motor cortex. Nature, 598(7879), 86-102.

Cho, C.-S. et al. (2021). Microscopic examination of spatial transcriptome using Seq-Scope. Cell, 184(13), 3559-3572.e22.

Dries, R. et al. (2021a). Advances in spatial transcriptomic data analysis. Genome Res., 31(10), 1706-1718

Dries, R. et al. (2021b). Giotto: a toolbox for integrative analysis and visualization of spatial expression data. Genome Biol., 22(1), 78.

Dyring-Andersen, B. et al. (2020). Spatially and cell-type resolved quantitative proteomic atlas of healthy human skin. Nat. Commun., 11(1), 5587.

Eng, C.-H. L. et al. (2019). Transcriptome-scale super-resolved imaging in tissues by RNA seqFISH. Nature, 568(7751), 235-239.

Fechner, S. and Goodman, M. B. (2018). Synaptic communication upon gentle touch. Neuron, 100(6), 1272-1274.

Geier, B. et al. (2020). Spatial metabolomics of in situ host-microbe interactions at the micrometre scale. Nat Microbiol, 5(3), 498-510.

Haanen, J. B. A. G. (2017). Converting cold into hot tumors by combining immunotherapies. Cell, 170(6), 1055-1056.

Hafemeister, C. and Satija, R. (2019). Normalization and variance stabilization of single-cell RNA-seq data using regularized negative binomial regression. Genome Biol., 20(1), 296.

Höskuldsson, A. (1988). PLS regression methods. J. Chemom., 2(3), 211-228

Hou, R. et al. (2020). Predicting cell-to-cell communication networks using NATMI. Nat. Commun., 11(1), 5011.

Janes, K. A. et al. (2005). A systems model of signaling identifies a molecular basis set for cytokine-induced apoptosis. Science, 310(5754), $1646-1653$

Liu, Y. et al. (2020). High-Spatial-Resolution Multi-Omics sequencing via deterministic barcoding in tissue. Cell, 183(6), 1665-1681.e18.

Longo, S. K. et al. (2021). Integrating single-cell and spatial transcriptomics to elucidate intercellular tissue dynamics. Nat. Rev Genet.

Marx, V. (2021). Method of the year: spatially resolved transcriptomics. Nat. Methods, 18(1), 9-14.

Nagai, J. S. et al. (2021). CrossTalkeR: Analysis and visualisation of ligand receptor networks. Bioinformatics.

Nutma, E. et al. (2020). Astrocyte and oligodendrocyte Cross-Talk in the central nervous system. Cells, 9(3).

Pelkmans, L. (2012). Cell biology. using cell-to-cell variability-a new era in molecular biology. Science, 336(6080), 425-426.

Regev, A. et al. (2017). The human cell atlas. Elife, 6

Rodriques, S. G. et al. (2019). Slide-seq: A scalable technology for measuring genome-wide expression at high spatial resolution. Science, 363(6434), 1463-1467.

Sapir, L. and Tzlil, S. (2017). Talking over the extracellular matrix: How do cells communicate mechanically? Semin. Cell Dev. Biol., 71, 99-105. Satija, R. et al. (2015). Spatial reconstruction of single-cell gene expression data. Nat. Biotechnol., 33(5), 495-502.

Seydel, C. (2021). Single-cell metabolomics hits its stride. Nat. Methods Sharpe, A. H. and Pauken, K. E. (2018). The diverse functions of the PD1 inhibitory pathway. Nat. Rev. Immunol., 18(3), 153-167. 
bioRxiv preprint doi: https://doi.org/10.1101/2022.01.12.476034; this version posted January 14, 2022. The copyright holder for this preprint (which was not certified by peer review) is the author/funder, who has granted bioRxiv a license to display the preprint in perpetuity. It is made available under a CC-BY-NC-ND 4.0 International license.

$$
\text { “output" — 2022/1/13 - 3:59 - page } 9 \text { — \#9 }
$$

Shimizu, K. et al. (2020). PD-1 imposes qualitative control of cellular transcriptomes in response to T cell activation. Mol. Cell, 77(5), 937950.e6.

Snijder, B. and Pelkmans, L. (2011). Origins of regulated cell-to-cell variability. Nat. Rev. Mol. Cell Biol., 12(2), 119-125.

Stickels, R. R. et al. (2021). Highly sensitive spatial transcriptomics at near-cellular resolution with Slide-seqV2. Nat. Biotechnol., 39(3), 313319.

Stuart, T. et al. (2019). Comprehensive integration of Single-Cell data. Cell, 177(7), 1888-1902.e21.

Svensson, V. et al. (2018). SpatialDE: identification of spatially variable genes. Nat. Methods, 15(5), 343-346.
Yamamoto, H. et al. (2014). Statistical hypothesis testing of factor loading in principal component analysis and its application to metabolite set enrichment analysis. BMC Bioinformatics, 15, 51.

Yuan, C. and Yang, H. (2019). Research on K-Value selection method of K-Means clustering algorithm. $J, 2(2), 226-235$.

Zhang, M. et al. (2021). Spatially resolved cell atlas of the mouse primary motor cortex by MERFISH. Nature, 598(7879), 137-143.

Zhu, J. et al. (2021). SPARK-X: non-parametric modeling enables scalable and robust detection of spatial expression patterns for large spatial transcriptomic studies. Genome Biol., 22(1), 184. 